\title{
A syndrome including thumb malformations, microcephaly, short stature, and hypogonadism
}

Joël Zlotogora, Judith Dagan, Adnan Ganen, Mazen Abu-Libdeh, Ziva Ben-Neriah, Tirza Cohen

\begin{abstract}
We report on eight patients from seven different families affected with a syndrome which includes thumb defects, short stature, microcephaly, and mental retardation. Most of the patients had additional malformations, in particular amenorrhoea and azoospermia in the adults. There were no haematological manifestations and the chromosomes were normal without evidence of breakage even after stimulation. In five of the cases the probands' mother received hormonal treatment before or at the beginning of her pregnancy or both.

The syndrome may be inherited as an autosomal recessive trait since the patients included both males and females and their parents were related in most cases. In addition, supporting this possibility, they all originated from a small village which may be considered as an isolate. However, in all cases but one, only one person was affected in each family and there was a significant apparent excess of healthy sibs of the probands. These observations may be the result of the variability of the syndrome or a more complex type of inheritance.

( $\mathcal{}$ Med Genet 1997;34:813-816)
\end{abstract}

Keywords: hypogonadism; Fanconi anaemia, microcephaly; thumb malformations

Department of Human Genetics, Hadassah University Hospital,

Hadassah Medical

School, Hebrew

University, Jerusalem,

Israel

J Zlotogora

J Dagan

Z Ben-Neriah

T Cohen

Kupat Holim Sick

Fund, Israel

A Ganen

M Abu-Libdeh

Correspondence to: Dr Zlotogora, Hadassah University Hospital, POB 12000 , Jerusalem il 91120 , Israel.

Received 14 January 1997 Revised version accepted for publication 12 May 1997
Congenital malformations of the thumbs are variable and often bilateral. They may include triphalangism, hypoplasia, aplasia, or polysyndactyly. In some cases the malformations are mild, such as short, large, or proximally placed thumbs. Malformations of the thumbs may be found together with other radial defects, such other malformations, often as part of known syndromes. ${ }^{1}$ Among the most frequent organs involved together with the thumbs are the heart, urinary tract system, skeleton, and blood. The VACTERL association includes radial ray deformities, defects of the vertebrae, anal atresia, tracheo-oesophageal fistula, renal malformations, and congenital heart defects. Many syndromes associated with thumb malformations are included in 113 entries in OMIM. $^{2}$ In some of the syndromes with absence of thumbs or radial rays or both, the major additional features are orofacial malformations, such as in Nager syndrome and Juberg-Hayward syndrome, or cardiovascular as hypoplasia or aplasia of the radius, or with malformations, such as in Holt Oram syndrome. In other syndromes blood dyscrasia is the pathognomonic feature, such as in Fanconi anaemia and the syndrome of thrombocytopenia and absent radius (TAR).

We report here on a group of patients presenting with thumb malformations together with microcephaly, mental retardation, short stature, and hypogonadism and discuss possible diagnosis and aetiology.

\section{Patients and methods}

All the patients originated from a small Muslim Israeli Arab village located near Jerusalem, fully integrated into the life of the State of Israel. Almost all the inhabitants can trace their ancestry back less than 10 generations from a very small number of founders. The village includes three large kindreds which each represent almost $25 \%$ of the household units (AR, JA, and IB) and three smaller kindreds (OT, AK, and SA). The inhabitants of the village are devout followers of their Islamic traditions and have maintained their isolation mainly by marriages within the family or within the village or both. In 1994, there were approximately 6000 inhabitants of the village.

Malformations of the thumbs were diagnosed in the genetic clinic at Hadassah Medical Center in seven patients originating from the village. In addition, a severely retarded female living in the village, never seen in the genetic clinic, was examined because of the similarity of her symptoms to those of the other patients. She had a proximal insertion of both thumbs with mild hypoplasia of the left thumb and therefore she was also included in the present study (OT F). A clinical summary of the eight patients is given in table 1 and the pedigree of the families in fig 1 .

The thumb malformations were variable, often different in the right and left thumb in the same patient as well as from patient to patient, and included aplasia, hypoplasia, and malposition. None of the patients had large or bifid thumbs, none had radial aplasia or hypoplasia, and their toes were normal. One patient was born with severe hydrocephalus with alobar holoprosencephaly and died in infancy. The other seven patients were affected with various degrees of mental retardation and had significant microcephaly (less than 2 SD for age). Four of the adult patients are mildly retarded, but integrated into daily life, and the other is severely retarded. Both adult males are working and one of them is married; the three adult females are single and helping at home. All but 


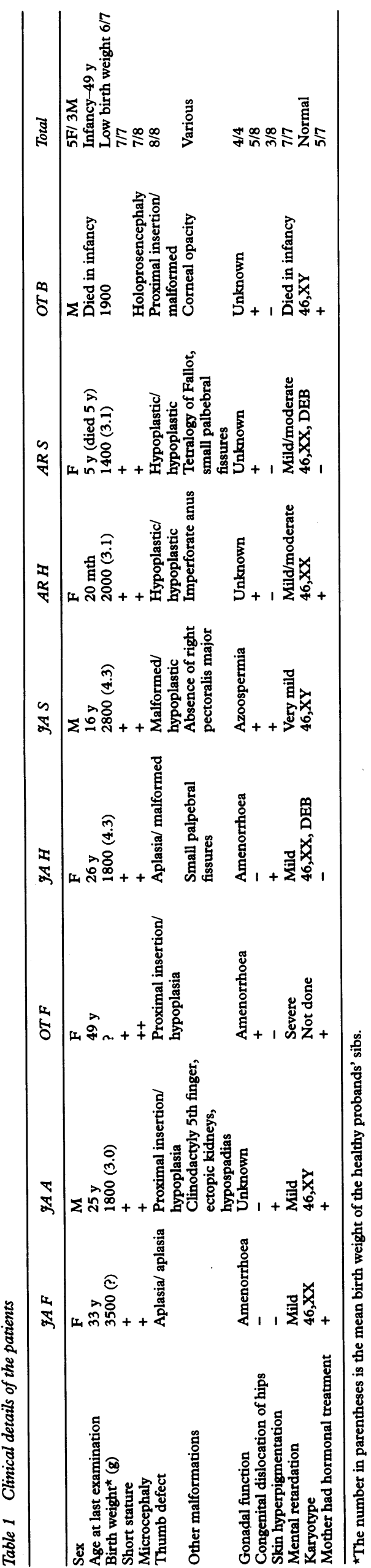

one patient had a low or very low birth weight; short stature was present in all the patients and the adults reached a height between 125 to 145 $\mathrm{cm}$. The three adult females had primary amenorrhoea. In one of them, an abdominal ultrasound was performed in which the ovaries were not seen. The male patient who was married was infertile because of azoospermia. None of the patients had any haematological problems and no malignancies have been diagnosed. In all the patients studied the chromosomes were normal and there was no induced chromosomal breakage after DEB stimulation performed in two unrelated patients.

Fertility problems were present in four of the patients' mothers. They received hormonal treatment (details on the type of treatment were not available to us) before and during the pregnancy of the affected patient. In three of these women the hormonal treatment was given only for the pregnancy of the proband; in the fourth, the mother also received treatment in another pregnancy from which a normal child was born. In a fifth family, the pregnancy was unwanted and the patient's mother took medication to have an abortion (JA F).

Three of the families were from the JA kindred (four patients), two from the AR kindred, and two from the OT kindred. In six out the seven families the probands' parents were closely related and in the last family the parents did not know of any relationship but originated from the same kindred (JA). Among the total of 40 children in the seven different families, there were two affected sibs in only one family (JA $\mathrm{H}$ and S). On the assumption that the syndrome was inherited as an autosomal recessive trait, eight of the 33 probands' sibs would have been expected to be affected instead of only one observed $\left(\chi^{2}=8.5, p<0.01\right)$.

\section{Discussion}

The similarity between the patients was evident, in particular when they were all examined over a short period of time, and we have no doubt that they are affected with the same syndrome. Among the numerous syndromes including thumb/radial malformations searched for in OMIM, LDDB, and POSSUM, most could be easily ruled out. In comparing those syndromes in which more than one family with affected patients was reported, the main differential diagnosis includes Fanconi anaemia and the Juberg-Hayward syndrome, since in both syndromes the major malformations overlap those present in our patients (table 2). All the malformations found in our patients have been reported in patients with Fanconi anaemia and the variability from one patient to another is similar in both syndromes. ${ }^{3}$ The major clinical differences are the rarity of mental retardation in Fanconi anaemia and the absence of bone marrow failure in our patients. It should be noted that recently two adult sibs affected with Fanconi anaemia were reported with microcephaly, hypogonadism, and mental retardation. ${ }^{4}$ The sister had no haematological manifestation or malignancy up to the age of 56 years, and Fanconi anaemia was diagnosed by the presence of 

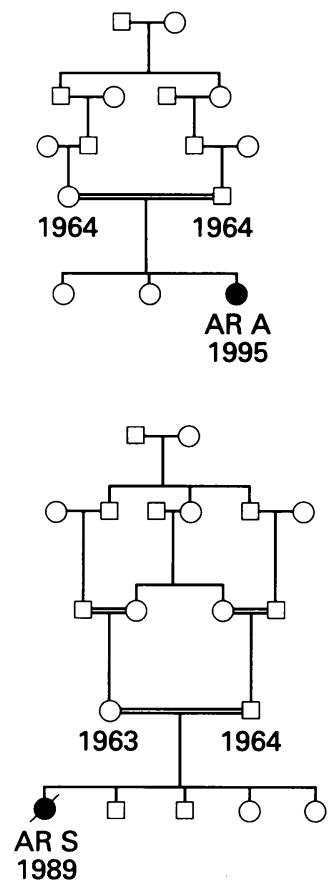

Figure 1 The pedigrees of the seven families. The affected children are identified by the same initials as in the tables. The date of birth is given under the symbols. A small triangle is used for spontaneous abortions and their number is indicated under the symbol. The pregnancies are indicated according to their order. In the families of $\mathcal{Y} A A$ and OT F, the parents did not remember the exact order of the spontaneous abortions after the birth of the proband.

chromosome breaks after mitomycin C stimulation. In our patients, however, the absence of chromosomal breakage after DEB stimulation excluded the diagnosis of Fanconi anaemia. Still, it may be speculated that the gene involved may be one of those causing Fanconi anaemia but with a different type of mutation or that it is a gene participating in a parallel developmental pathway. The Juberg-Hayward syndrome has been reported in relatively few patients up to now and includes the major malformations found in our patients: thumb malformations, growth retardation, microcephaly, and mental retardation (table 2). ${ }^{5}$ The patients with the Juberg-Hayward syndrome were young and no data were available on their gonadal function. The major differences were the absence of skin hyperpigmentation and congenital dislocation of hips in the patients with the Juberg-Hayward syndrome and the absence of characteristic facial features in our patients, in particular the cleft lip/palate. At this stage the possibility that the syndrome

Table 2 Comparison of the features of the syndrome with those of Fanconi anaemia and the fuberg-Hayward syndrome

\begin{tabular}{llcc}
\hline & $\begin{array}{l}\text { Fanconi } \\
(\%)\end{array}$ & $\begin{array}{l}\text { fuamia } \\
\text { fundg-Hayward }\end{array}$ & $\begin{array}{l}\text { Our patients } \\
\text { syndrome }\end{array}$ \\
\hline Growth retardation & 77 & $9 / 9$ & $6 / 7$ \\
Skin hyperpigmentation & 73 & - & $3 / 8$ \\
Hypogenitalia & 51 & - & $4 / 4$ \\
Combined radial ray deformities & 66 & $6 / 9$ & $8 / 8$ \\
Thumb anomaly, absence & 55 & $6 / 9$ & $8 / 8$ \\
Hypoplastic/absent radius & 13 & $5 / 9$ & - \\
Microcephaly & 37 & $7 / 9$ & $7 / 8$ \\
Renal anomalies & 32 & $3 / 8$ & $1 / 8$ \\
Mental retardation & 29 & $1 / 3$ & $7 / 7$ \\
Ear anomaly & 11 & $2 / 3$ & - \\
Mild microphthalmia & 14 & - & $2 / 8$ \\
Cardiac murmur & 29 & - & $1 / 8$ \\
CDH & 9 & - & - \\
Pancytopenia & + & - & - \\
Chromosomal breakage & + & + & - \\
Cleft lip/palate, typical facial features & - & & $5 / 8$ \\
\hline
\end{tabular}

reported here is the Juberg-Hayward syndrome cannot be completely ruled out, in particular since most of the facial features are subtle and probably variable in different ethnic backgrounds. In addition, since only a few patients have been reported, the full spectrum of malformations which may be present in the Juberg-Hayward syndrome is still unknown.

All the patients originated from a small village which may be considered almost as an isolate. In the village most of the marriages are by preference within the family and almost always within the larger kindred. Indeed the patients' parents were closely related in six out of the seven families. In addition, the patients included males and females and their parents were all healthy. All these observations are in favour of autosomal recessive inheritance. However, only one of the 33 sibs of the probands was affected, which is significantly different from that expected in an autosomal recessive disorder. A possible explanation may be that some of the sibs have mild features because of the variability of the syndrome. However, even though we could not examine most of the patients' sibs for this study, they are under the medical supervision of the authors working in the village. No major malformations are known and all the married sibs are fertile. Another possibility may be that the syndrome has a more complex inheritance. An observation which may be in favour of such a possibility is that five of the seven probands' mothers had hormonal treatment at the beginning of the pregnancy. This may be particularly relevant since in all cases but one the treatment was given only during the pregnancy of the affected child. It can be envisaged that the risk of developing the syndrome exists both for homozygotes and heterozygotes of an unknown 
gene mutation, the possibility of developing the clinical syndrome being high in the people who inherit two copies of the abnormal gene but also existing in the heterozygotes. Heterozygotes will be affected only if additional factors are present, such as hormonal treatment at the beginning of the pregnancy. A similar situation has been reported which explains the relatively high frequency of Hirschsprung disease among the Mennonites. ${ }^{6}$ In this isolate, both homozygotes and heterozygotes for a mutation in the endothelin-B receptor gene have a high risk of being affected with Hirschsprung disease. The risk is higher for the homozygotes (74\%) than for the heterozygotes $(21 \%)$ since the mutation is only one of the risk factors for the development of Hirschsprung disease.

1 Temtamy S, McKusick V. The genetics of hand malformations. Birth Defects 1978;14:36-184.

On Line Mendelian Inheritance in Man (TM). Center for Medical Genetics, Johns Hopkins University (Baltimore, MD) and National Center of Biotechnology Information, National Library of Medicine (Bethesda, MD), 1997.

3 Glanz A, Fraser CF. Spectrum of anomalies in Fanconi anaemia. $\mathcal{F}$ Med Genet 1982;19:412-16.

4 Kwee ML, van der Kleij JM, van Essen AJ, et al. An atypical case of Fanconi anemia in eldery siblings. Am $\mathcal{F}$ Med Genet 1997;68:362-6.

5 Verloes A, Le Merrer M, Davin JC, et al. The orocraniodigital syndrome of Juberg and Hayward. $\mathcal{F}$ Med Genet ital syndrome

6 Puffenberger EG, Hosoda K, Washington SS, et al. A missense mutation of the endothelin-B receptor gene in
multigenic Hirschsprung disease. Cell 1994;79:1257-66. 